\title{
LFchimera protects HeLa cells from invasion by Yersinia spp. in vitro
}

\author{
Tjitske Sijbrandij • Antoon J. Ligtenberg - Kamran Nazmi • Petra A. M. van den Keijbus • \\ Enno C. I. Veerman · Jan G. M. Bolscher • Floris J. Bikker $\mathbb{1}$
}

Received: 30 May 2018/Accepted: 17 August 2018/Published online: 22 August 2018

(C) The Author(s) 2018

\begin{abstract}
Yersinia pestis is the causative agent of plague. As adequate antibiotic treatment falls short and currently no effective vaccine is available, alternative therapeutic strategies are needed. In order to contribute to solving this problem we investigated the therapeutic potential of the peptide construct LFchimera against the safer-to-handle $Y$. pestis simulants Yersinia enterocolitica and Yersinia pseudotuberculosis in vitro. LFchimera is a heterodimeric peptide construct mimicking two antimicrobial domains of bovine lactoferrin, i.e. lactoferrampin and lactoferricin. LFchimera has been shown to be a potent antimicrobial peptide against a variety of bacteria in vitro and in vivo. Also $Y$. enterocolitica and $Y$. pseudotuberculosis have been shown to be susceptible for LFchimera in vitro. As Yersiniae spp. adhere to and invade host cells upon infection, we here investigated the effects of LFchimera on these processes. It was found that LFchimera has the capacity to inhibit hostcell invasion by Yersiniae spp. in vitro. This effect appeared to be host-cell mediated, not bacteriamediated. Furthermore it was found that exposure of
\end{abstract}

T. Sijbrandij · A. J. Ligtenberg - K. Nazmi ·

P. A. M. van den Keijbus - E. C. I. Veerman .

J. G. M. Bolscher · F. J. Bikker ( $₫)$

Department of Oral Biochemistry, Academic Centre for

Dentistry Amsterdam, University of Amsterdam and VU

University Amsterdam,

Gustav Mahlerlaan 30041081 LA Amsterdam, The

Netherlands

e-mail: f.bikker@acta.nl human HeLa epithelial cells to both LFchimera and the bacterial strains evoked a pro-inflammatory cytokine release from the cells in vitro.

Keywords Antimicrobial peptide - Biowarfare simulants · Cellular adhesion and invasion .

Lactoferrin · LFchimera

\section{Introduction}

Yersinia pestis is the causative agent of plague. Its strategy for transmission relies on the colonization of rat fleas. Once inside the human host, Y. pestis can cause bubonic, pneumonic, and septicaemic plague with mortality rates approaching $100 \%$ in absence of antibiotic treatment. The Centers for Disease Control and Prevention (CDC) classifies $Y$. pestis as a category A biological warfare agent (BWA) (Jansen et al. 2014; Rotz et al. 2002). In order to overcome several safety, security and logistical drawbacks in biomedical investigations on $Y$. pestis research is often performed using it safer simulants Yersinia enterocolitica and Yersinia pseudotuberculosis (Adducci et al. 2016; Bikker et al. 2006; Dawson and Liu 2008; Kaman et al. 2011; Sijbrandij et al. 2017). Y. enterocolitica and $Y$. pseudotuberculosis can cause yersiniosis, an infectious disease which generally lasts for one to three weeks (Galindo et al. 2011). Infection can occur by the 
consumption of undercooked meat, unpasteurized milk or contaminated water. Common symptoms include fever, abdominal pain, and in children diarrhea, which can be bloody in severe cases. Complications are rare, and may include skin rash, joint pains, ileitis, erythema nodosum, septicemia, and acute arthritis (Bancerz-Kisiel and Szweda 2015).

Since most of the symptoms caused by $Y$. enterocolitica and $Y$. pseudotuberculosis are self-limiting, specific antibiotic treatment is generally not needed, especially in relative mild cases. For example, patients with dehydration from gastroenteritis are given supportive therapy, including treatment aimed at replacing fluids. Antibiotics are indicated, however, for those patients who develop more severe infections, such as septicemia, or who develop infections at specific sites, such as bone. But, in case of plaguecaused by $Y$. pestis-antibiotic treatment must be started immediately without laboratory confirmation to avoid the risk of transmission and mortality. Antibiotics generally used for treatment include aminoglycosides and trimethoprim-sulfamethoxazole. Also third-generation cephalosporins, tetracyclines and fluoroquinolones can be applied, yet with limited use in children. Although these antibiotics are still used, resistance is emerging (Guiyoule et al. 1997, 2001; Hernandez et al. 2003; Hinnebusch et al. 2002). Besides, as currently no effective and licensed vaccine is available for the prevention of plague (Oyston and Williamson 2013; Verma and Tuteja 2016) alternative strategies to develop therapies against Yersinia related infections are needed.

LFchimera is a heterodimeric peptide construct designed to mimic two antimicrobial domains, Lactoferricin (LFcin) and Lactoferrampin (LFampin), which are present in the N1-domain of bovine lactoferrin (bLF) and are responsible for its antimicrobial activity in situ (Bolscher et al. 2009, 2012). LFchimera has been shown to be a potent antimicrobial peptide against a variety of bacteria (Bolscher et al. 2009) in vitro and in vivo, including Staphylococcus aureus (Flores-Villaseñor et al. 2010), Escherichia coli O157:H7 (Flores-Villaseñor et al. 2012a), Streptococcus pneumoniae (León-Sicairos et al. 2014) and Burkholderia pseudomallei (Kanthawong et al. 2009; Puknun et al. 2013, 2016), the latter being classified by the CDC as a category B biological warfare agent (BWA) (Rotz et al. 2002). Also $Y$. enterocolitica and $Y$. pseudotuberculosis have been shown to be susceptible to LFchimera (Sijbrandij et al. 2017). Overall, the bactericidal activity of the LFchimera is related to membrane disturbing effects, i.e. membrane permeabilization and depolarization.

During infection, Yersiniae first adhere to host cells, followed by invasion. So, hypothetically, inhibition of cell adhesion and invasion would contribute to the therapeutic effect of the LF peptides, on top of their antimicrobial activity. Therefore, the aim of the presented study was to map the effects of bovine LF (bLF) derived peptides on adhesion and invasion of $Y$. enterocolitica and $Y$. pseudotuberculosis. For this study it was chosen to use human HeLa epithelial cells as the in vitro model system, as Hela cells or HeLaderived cells were used in literature before for Yersinia studies (Dersch and Isberg 1999; Di Biase et al. 2004; Schulte et al. 1996, 2000).

\section{Materials and methods}

bLF and LF derived peptides

bLF (20\% iron saturated) was kindly provided by DMV International (Veghel, The Netherlands). LF derived peptides (Table 1) were synthesized by solid phase peptide synthesis using Fmoc chemistry with a Siro II synthesizer (Biotage, Uppsala, Sweden) according to the manufacturer's protocol. Purification by Reverse Phase-HPLC was conducted as described previously (Bolscher et al. 2009). Identity of the peptides was confirmed by mass spectrometry (Bruker Daltonik GMBH, Bremen, Germany) and molar concentrations were calculated based on their weight.

Bacterial strains, cell line and culture conditions

Yersinia enterocolitica (DSM 4780) and Y. pseudotuberculosis (DSM 8992) were cultured overnight aerobically in trypticase soy broth (TSB) medium at $30{ }^{\circ} \mathrm{C}$ and colony forming units (CFU) were determined on trypticase soy agar (TSA) as described earlier (Sijbrandij et al. 2017). Human HeLa epithelial cells were grown in RPMI1640 medium (RPMI, Life Technologies, Carlsbad, USA) supplemented with $10 \%$ fetal bovine serum (HyClone, South Logan, USA) and $1 \%$ penicillin/streptomycin/amphotericin B (Sigma-Aldrich, St. Louis, USA) at $37{ }^{\circ} \mathrm{C}$ with $5 \%$ $\mathrm{CO}_{2}$. 
Table 1 Sequences and characteristics of the peptides investigated

\begin{tabular}{|c|c|c|c|c|}
\hline \multirow[t]{2}{*}{ Peptide $^{a}$} & \multirow[t]{2}{*}{ Primary structure } & \multirow[t]{2}{*}{ Charge } & \multicolumn{2}{|c|}{$\operatorname{MBC}(\mu \mathrm{M})^{\mathrm{b}}$} \\
\hline & & & Y. e. & $Y . p$. \\
\hline LFampin265-284 & DLIWKLLSKAQEKFGKNKSR & +4 & 6.3 & ND \\
\hline LFcin $17-30$ & FKCRRWQWRMKKLG & +6 & 0.8 & ND \\
\hline LFchimera $^{c}$ & $\begin{array}{r}\text { DLIWKLLSKAQEKFGKNKSR } \\
\text { FKCRRWQWRMKKLG-K }\end{array}$ & +12 & 0.2 & 1.6 \\
\hline LFampin265-284 and LFcin17-30 & $\begin{array}{l}\text { DLIWKLLSKAQEKFGKNKSR } \\
\text { and FKCRRWQWRMKKLG }\end{array}$ & +10 & 0.8 & ND \\
\hline
\end{tabular}

Y. e.: Y. enterocolitica, Y. p.: Y. pseudotuberculosis

${ }^{a}$ The purity of the peptides was at least $95 \%$ and the authenticity of the peptides was confirmed by MALDI-TOF mass spectrometry

${ }^{\mathrm{b}}$ Minimal bactericidal concentration (MBC), in $1 \mathrm{mM}$ phosphate buffer (from Sijbrandij et al. 2017). ND: not detected up to $50 \mu \mathrm{M}$ peptide

${ }^{\mathrm{c}}$ A single C-terminal lysine amide $(\mathbf{K})$ was substituted at the $\alpha$-and $\varepsilon$-amino groups with the two peptides via the C-terminal site, while leaving the two $\mathrm{N}$-termini as free ends

Bactericidal activity of LF peptides

The killing activities of the LF peptides against the Yersinia spp. were determined as described previously with the exception that the incubation was performed in RPMI (Sijbrandij et al. 2017). In this way conditions were the same as for cytotoxicity determinations and adhesion and invasion assays (see below). Briefly, bacterial cells were washed three times and approximately $2 \times 10^{6} \mathrm{CFU} / \mathrm{ml}$ were re-suspended in RPMI medium. The bacterial suspension was then added to an equal volume of the tested agents $(0.1-50 \mu \mathrm{M}$ final concentration). A bacterial suspension in RPMI without peptide served as a control. Following incubation at $37{ }^{\circ} \mathrm{C}$ for $60 \mathrm{~min}$, the mixture was serially diluted in a physiological concentration of saline and plated in triplicate on TSA. After $24 \mathrm{~h}$ of incubation at $37{ }^{\circ} \mathrm{C}$ colonies were counted. A bactericidal effect was defined as a $\geq 3 \log _{10}$ reduction in CFU/ml compared with the initial inoculum.

Cytotoxicity of bLF and LF peptides on HeLa cells

Cytotoxicity of bLF and the LF peptides toward HeLa cells was analyzed by monitoring mitochondrial activity. For this, approximately $5 \times 10^{4} \mathrm{HeLa}$ cells were seeded in a 96-well plate and cultured serum-free for $16 \mathrm{~h}$. Cells were washed twice with PBS. A serial dilution of bLF and each peptide was made in PBS $(0-25 \mu \mathrm{M})$. Cells we were incubated at $37{ }^{\circ} \mathrm{C}$ under
$5 \% \mathrm{CO}_{2}$ for $1 \mathrm{~h}$. Maximal cytotoxicity was induced by adding 0.1\% Triton X-100 (Sigma-Aldrich, St. Louis, MO, USA). Cells were washed twice with PBS and incubated in PBS containing $0.5 \mathrm{mg} / \mathrm{ml} 3-(4,5-$ dimethylthiazol-2-yl)-2,5-diphenyltetrazolium bromide (MTT, ThermoFisher Scientific, Bleiswijk, the Netherlands) for $2 \mathrm{~h}$ and washed again. The MTTcrystals that were precipitated in the cells were resuspended in 100\% dimethylsulfoxide (DMSO, Sigma-Aldrich). Absorption was measured at $570 \mathrm{~nm}$ with $630 \mathrm{~nm}$ for background correction using a Multiscan FC microplate photometer (ThermoFisher Scientific,). In parallel, cytotoxic effects of bLF and the peptides on Hela cells were analyzed by measuring the lactate dehydrogenase (LDH) release using an LDH-cytotoxicity kit (Abcam, Cambridge, UK) according to the manufacturer's instructions ( $\mathrm{Si}$ jbrandij et al. 2017). The experiments were performed in duplicate and repeated three times.

Effects of LF peptides on bacterial adhesion and invasion

The effect of LF peptides on adhesion and invasion of the Yersinia spp. were carried out essentially as described previously (Wang et al. 2008; Tan et al. 2017). Briefly, HeLa cells were plated at a density of $1 \times 10^{5}$ cells in 24-wells plates (Greiner, Recklinghausen, Germany). After attachment the LF peptides were added at concentrations up to $3.1 \mu \mathrm{M}$. After $1 \mathrm{~h}$, 
the unbound peptides were removed by washing three times using PBS. Fresh stocks of bacteria were harvested by centrifugation at $10,000 \times g$ for $10 \mathrm{~min}$, washed twice in PBS and resuspended in PBS to a density of $5 \times 10^{8}$ cells $/ \mathrm{ml}$. Next, bacteria were added to the HeLa cells at a multiplicity of infection (MOI) of $100 \mathrm{CFU} / \mathrm{cell}$. Subsequently, the 24-wells culture plates were centrifuged at $300 \times g$ for $10 \mathrm{~min}$ to promote contact between bacteria and HeLa cells and then incubated at $37{ }^{\circ} \mathrm{C}$ with $5 \% \mathrm{CO}_{2}$ for $2 \mathrm{~h}$ and washed three times with PBS to remove unadhered bacteria. To determine the number of cell-associated bacteria, this solutions was serially diluted in a physiological concentration of saline and plated in triplicate on TSA and colonies were counted after $24 \mathrm{~h}$ of incubation at $37{ }^{\circ} \mathrm{C}$ as described previously (Sijbrandij et al. 2017).

To determine the number of intracellular bacteria, extracellular bacteria were killed by treatment of $100 \mu \mathrm{g} / \mathrm{ml}$ gentamicin in fresh medium during $2 \mathrm{~h}$. The cells were further cultured at $37{ }^{\circ} \mathrm{C}$ with $5 \% \mathrm{CO}_{2}$ for $4 \mathrm{~h}$, washed three times with PBS and lysed by the addition of $0.1 \%$ Triton $\mathrm{X}-100$ at $37{ }^{\circ} \mathrm{C}$ with $5 \% \mathrm{CO}_{2}$ for $20 \mathrm{~min}$. Again, this solutions was serially diluted in a physiological concentration of saline and plated in triplicate on TSA and colonies were counted after $24 \mathrm{~h}$ of incubation at $37{ }^{\circ} \mathrm{C}$ as described above. The number of cell-associated bacteria subtracted by the number of intracellular bacteria was used as measure for adhesion.

As control for treatment timing, LFchimera up to $3.1 \mu \mathrm{M}$ was added for $1 \mathrm{~h}$ post infection, before or after gentamicin treatment in the above described protocol. Furthermore, the effect of preincubation of bacteria or preincubation of HeLa cells with $3.1 \mu \mathrm{M}$ LFchimera at $37^{\circ} \mathrm{C}$ for $1 \mathrm{~h}$ were tested similarly.

\section{Effect of LFchimera on cytokine release}

The effect of LFchimera on the release of cytokine IL6 and chemokine IL-8 from HeLa cells upon infection with the Yersina spp. were analyzed using in essence the experimental set-up as described above. Briefly, HeLa cells were plated at a density of $2 \times 10^{5}$ cells in 24-wells plates. Fresh stocks of bacteria were harvested by centrifugation at $10,000 \times g$ for $10 \mathrm{~min}$, washed $3 \times$ in PBS and resuspended in serum-free medium to a density of $5 \times 10^{8}$ cells $/ \mathrm{ml}$. Next, the HeLa cells were washed twice with PBS and medium was added with $0,1.6$ or $3.1 \mu \mathrm{M}$ LFchimera. The cells were incubated for $1 \mathrm{~h}$ washed twice with PBS and bacteria were added to the HeLa cells at a multiplicity of infection (MOI) of $100 \mathrm{CFU} /$ cell and centrifuged at $300 \times g$ for $10 \mathrm{~min}$ to promote contact between bacteria and HeLa cells. After incubated for $2 \mathrm{~h}$ at $37^{\circ} \mathrm{C}$, $5 \% \mathrm{CO}_{2}$ the 24-wells culture plates were washed three times with PBS to remove unadhered bacteria and further incubated in $0.5 \mathrm{ml}$ serum free medium for $24 \mathrm{~h}$. Subsequently, the concentrations of IL- 6 and IL8 were determined in $100 \mu \mathrm{l}$ samples from the 24-wells culture plates after remove the bacteria by centrifuged at $10,000 \times g$ for $5 \mathrm{~min}$, using the PeliKine compact human IL-6 and IL-8 ELISA kits according to the manufacturer's protocol (Sanquin, Amsterdam, The Netherlands).

Statistical analysis

Statistical analysis was performed using a one-way ANOVA and posthoc Bonferroni test using SPSS Statistics for Windows version 20.0 (IBM Corp, Armonk, NY, USA). $P$ values $<0.05$ were considered statistical significant.

\section{Results}

Bactericidal activity of LF peptides

Before exploring the potential effect of the LF peptides on adhesion and invasion of the Yersinia spp., anti-bacterial activity of these peptides in HeLa cell culture medium was measured, to ensure that subsequent experiments are performed at sub-bactericidal levels. Under these conditions both LFcin17-30 and LFampin265-284 as well as the combination of the two peptides had no bactericidal effect on the Yersinia spp. (Fig. 1). Only the LFchimera, exhibited inhibitory activities in a dose response manner. LFchimera at a concentration $3.1 \mu \mathrm{M}$ caused ${ }^{10} \log =1$ reduction of $Y$. enterocolitica increasing to almost $3 \log _{10}$ reduction at higher concentrations up to $50 \mu \mathrm{M}$. Y. pseudotuberculosis was a little more sensitive as $3.1 \mu \mathrm{M}$ LFchimera induced a ${ }^{10} \log >2$ reduction in CFU count, being close to the maximal effect of less than $3 \log _{10}$ with $50 \mu \mathrm{M}$ (Fig. 1). However, in contrast to the bactericidal activity at low ionic strength (Table 1), the anti-bacterial effect of 


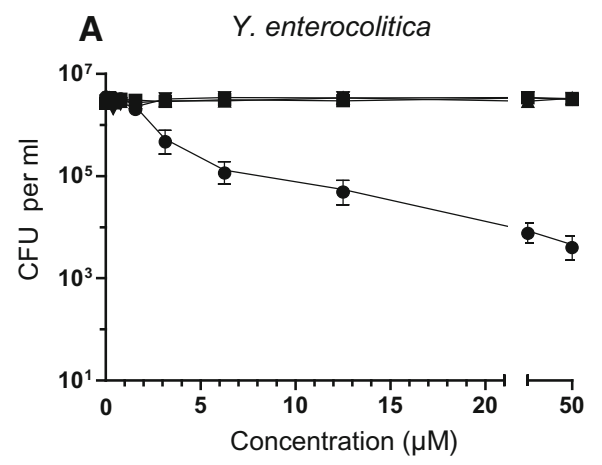

B Y. pseudotuberculosis

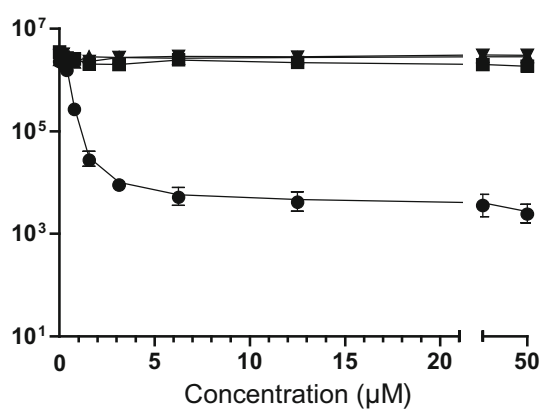

$\nabla$ LFcin17-30 $\Delta$ LFampin265-284

Fig. 1 Bactericidal activity of bLF-peptides on $Y$. enterocolitica (a) and Y. pseudotuberculosis (b) determined in RPMI cell culture medium after an incubation of $1 \mathrm{~h}$. Bactericidal effect

LFchimera in cell culture medium remained below the ${ }^{10} \log =3$ reduction in CFU counts, used as cut-off value for bactericidal effect (Sijbrandij et al. 2017). Therefore the concentration of $3.1 \mu \mathrm{M}$ LFchimera in HeLa cell culture medium was considered to be a sublethal dose towards the Yersinia spp. used.

Cytotoxicity of bLF and the LF peptides

To prevent using cytotoxic levels of the LF peptides in subsequent experiments, cytotoxicity toward HeLa cells was determined by measuring leakage of LDH as well as by monitoring mitochondrial activity (Fig. 2). In both assays LFcin17-30 and LFampin265-284 as well as the combination showed no or only minor
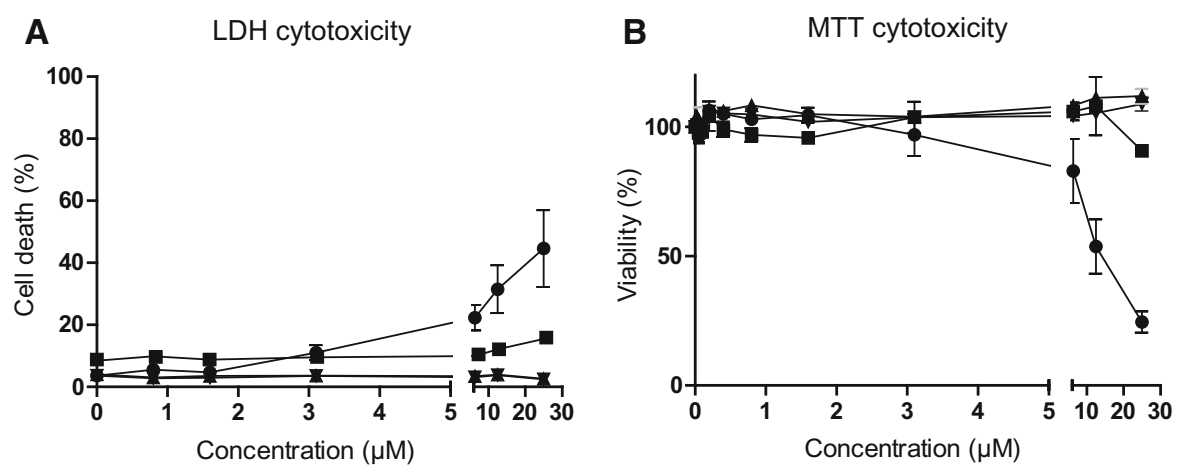

$$
\nabla \text { LFcin17-30 } \Delta \text { LFampin265-284 }
$$

Fig. 2 HeLa cell cytotoxicity of bLF-peptides determined in RPMI medium after an incubation of $1 \mathrm{~h}$ : LDH-leakage as measure for cell death (a) and MTT assay as measure for was defined as a ${ }^{10} \log$ [reduction] $>3$ in viability. Data are shown as mean values \pm SEM from three independent experiments carried out in triplicate

effect up to $25 \mu \mathrm{M}$ concentrations. The first sign of any cytotoxic effect was found at LFchimera concentrations of $6.1 \mu \mathrm{M}$. Therefore peptide concentrations up to $3.1 \mu \mathrm{M}$ were considered to be not cytotoxic and were used to analyze the effect on the adhesion and invasion of the Yersinia spp. bLF did not show any cytotoxic effect up to $25 \mu \mathrm{M}$ (data not shown).

Adhesion and invasion

None of the LF peptides at a concentration up to $3.1 \mu \mathrm{M}$ showed any effect on the adherence of both $Y$. enterocolitica and $Y$. pseudotuberculosis to the HeLa cells (Fig. 3). Strikingly, invasion of both Yersinia spp. was exclusively inhibited by LFchimera at

B

- LFcin \& LFampin • LFchimera

viability (b). Data are shown as mean values \pm SEM from three independent experiments carried out in triplicate 
Fig. 3 Effects of bLFpeptides on adhesion and invasion of $Y$. enterocolitica (a), Y. pseudotuberculosis (b), Y. enterocolitica (c), and $Y$. pseudotuberculosis (d) on HeLa epithelial cells treated for $1 \mathrm{~h}$ with nonlethal concentrations of peptides. Data are shown as mean values \pm SEM from three independent experiments carried out in triplicate
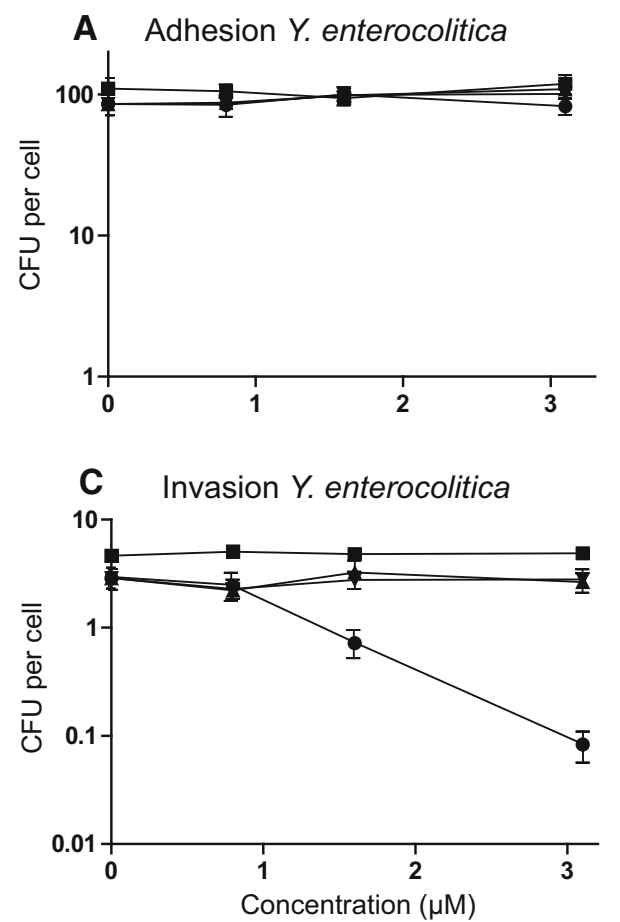

$\nabla$ LFcin17-30 \ LFampin265-284
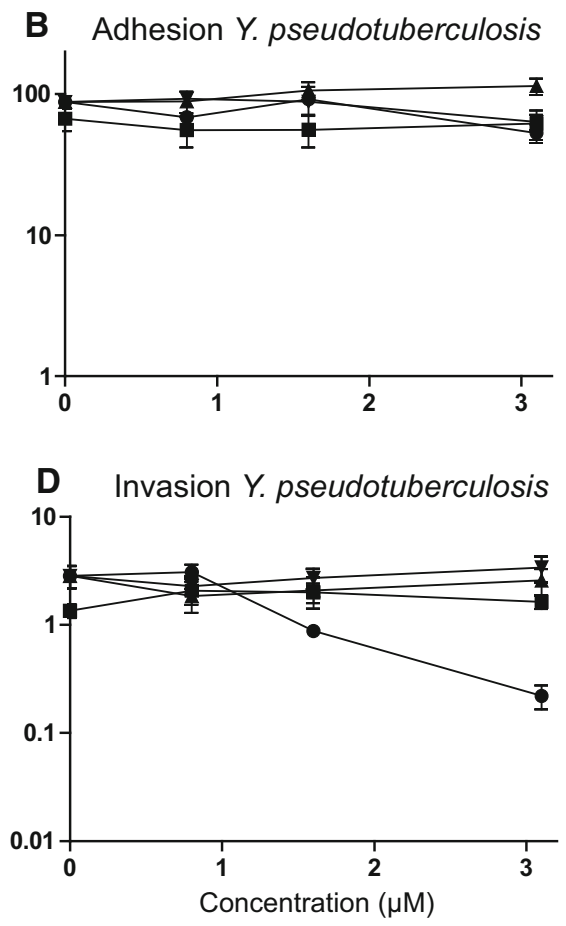

- LFcin \& LFampin - LFchimera concentrations as low as $1.6 \mu \mathrm{M}$. At a concentration of $3.1 \mu \mathrm{M}$ LFchimera, the invasion of $Y$. enterocolitica was inhibited about twice as much as $Y$. pseudotuberculosis (30-fold and 15-fold respectively, Fig. 3). In contrast, LFcin, LFampin and the combination of the two peptides had no effect on the capacity of the Yersinia spp. to invade the HeLa cells.

Next, we determined whether the inhibition was mediated through an effect on the Yersinia spp. or on the HeLa cells. Pretreatment of the Yersinia spp. with 3.1 $\mu \mathrm{M}$ LFchimera had no effect on the invasion whereas pretreatment of the HeLa cells significantly reduced the invasion capacity of both Yersinia spp. by circa $80 \%$ of the level of invasion without LFchimera (Fig. 4). Control experiments in which LFchimera was added post infection, before or after gentamicin treatment indicated that the LFchimera did not made the cells permeable to gentamicin nor killed the bacteria intracellularly (data not shown).

Cytokine and chemokine release

Because LFchimera inhibited invasion of the Yersinia spp. through an effect on the target cells, we

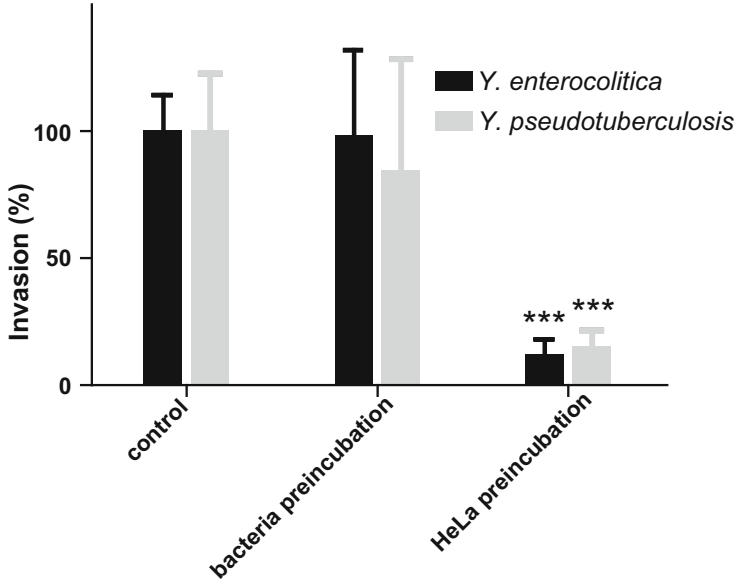

Fig. 4 Inhibition of invasion by $3.1 \mu \mathrm{M}$ LFchimera after preincubated with either bacteria or HeLa cells for $1 \mathrm{~h}$. Invasion is presented as percentage of the controls without LFchimera. Data are shown as mean values \pm SD from three independent experiments carried out in triplicate. Asterisk indicates statistical significant differences versus control incubations $(* * * \mathrm{P}<0.0005)$

investigated whether the HeLa cells released inflammatory mediators, such as IL-6 and IL-8, upon infection with bacteria. Indeed, an almost fourfold 
increase in the release of IL-8 by Yersinia-infected HeLa cells was found in the presence of $3.1 \mu \mathrm{M}$ LFchimera (Fig. 5). The release of IL-6 was also increased, be it that LFchimera also stimulated the IL6 production of uninfected HeLa cells to some extent (Fig. 5).

\section{Discussion}

In the present paper we show and characterize the effects of LFchimera on the capacity of Y. enterocolitica and $Y$. pseudotuberculosis to adhere to-, and invade $\mathrm{HeLa}$ cells. Previously it was found that in $1 \mathrm{mM}$ potassium phosphate buffer (PPB), LFchimera exhibited bactericidal activity at concentrations as low as 0.2 and $1.6 \mu \mathrm{M}$ towards the Yersinia spp., respectively (Table 1; Sijbrandij et al. 2017). In contrast, in RPMI medium, used to culture HeLa cells, the antiYersinia activity of LFchimera was only found at much higher concentrations (Fig. 1). Though LFchimera had different antimicrobial effect on the individual species, at concentrations that are sub-lethal and not cytotoxic in RPMI medium, i.e. up to $3.1 \mu \mathrm{M}$, LFchimera inhibited the capacity of the Yersinia spp. to invade HeLa cells (Fig. 1). Inhibition of invasion could be induced by pre-incubation of the HeLa cells but not by pre-incubation of the bacteria. As reference, the native bLF showed no bactericidal and cytotoxic activity in vitro up to $50 \mu \mathrm{M}$ and $25 \mu \mathrm{M}$, respectively. Therefore, the study subsequently focused on the LF peptides only.
In line with this study invasion of Escherichia coli (EHEC) O157:H7 was found to be inhibited by LFchimera in vivo. BALB/c mice inoculated intragastrically with EHEC O157:H7 showed chronic intestinal infection with the pathogen that persisted over 6 days and resulted in a high mortality rate (90\%). LFchimera could significantly decrease this mortality rate by $50 \%$ (Flores-Villaseñor et al. 2012a). Moreover, it was found that LFchimera inhibited invasion of enteropathogenic E. coli to Hep-2 cells in vitro (Flores-Villaseñor et al. 2012b). Also LFcin17-41, which is an extended variant of the LFcin17-30 was found to inhibit internalization of invasion-expressing E. coli (Longhi et al. 1994). Besides, LFcin17-41 was found to inhibit internalization of $Y$. enterocolitcia and $Y$. pseudotuberculosis in vitro (Di Biase et al. 2004). In our study however, LFcin17-30 showed no effect on adhesion and invasion of HeLa cells by the Yersinia spp. The additional 11 amino acids in LF17-41 force LFcin174 into a $\beta$-sheet conformation and appear to be crucial for this characteristic (Hwang et al. 1998). In contrast, LFcin17-30 conducts partially an $\alpha$-helix conformation (Haney et al. 2012). Moreover, the parent protein bLF did not affect Pseudomonas aeruginosa adhesion to primary bronchial epithelium from a cystic fibrosis (CF) patient but significantly reduced the number of intracellular bacteria (Frioni et al. 2014). This bLF effect was attributed to a reduction of the intracellular bacterial survival. However, in our study the LFchimera inhibited invasion (Figs. 3, 4) while having no effect on the intracellular survival (data not shown).
Fig. 5 Effects of $3.1 \mu \mathrm{M}$ LFchimera on IL-8 (a) and IL-6 (b) expression upon infection with and without $Y$. enterocolitica and $Y$. pseudotuberculosis. Data are shown as mean values \pm SD from three independent experiments carried out in triplicate. Asterisk indicates statistical significant differences versus the incubations without LFchimera $(* \mathrm{P}<0.05 ; * * \mathrm{P}<0.005)$
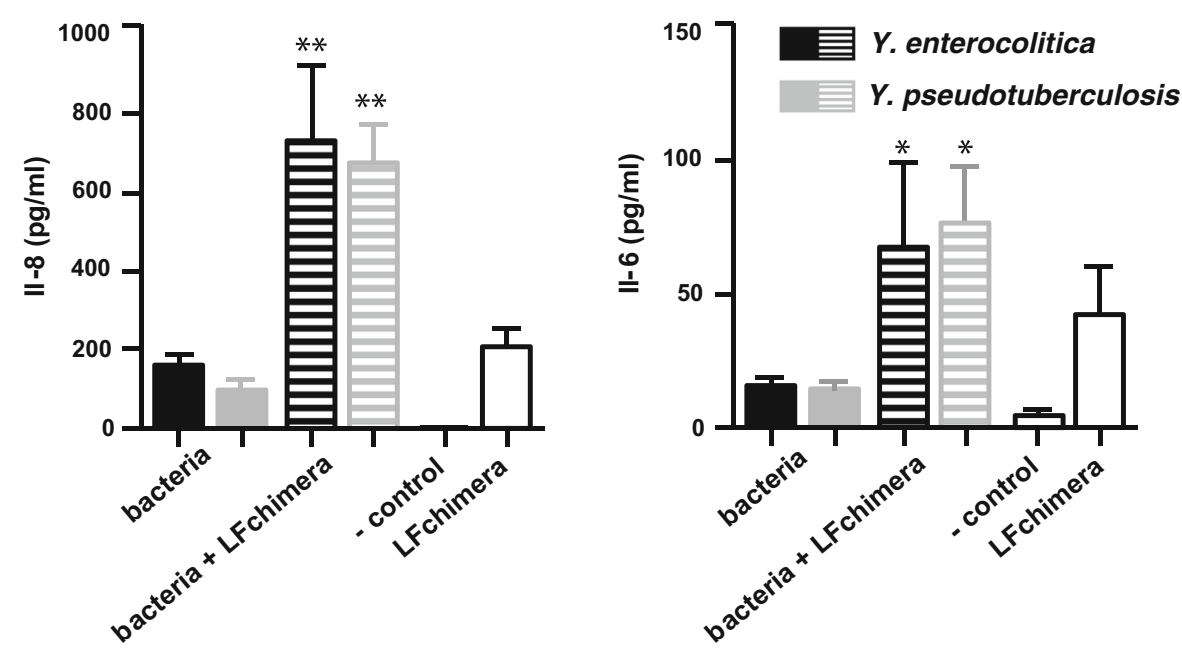
Although this study primarily focused on the effect of the LFchimera on adhesion and invasion some speculations can be made on the molecular mechanisms of the inhibition of invasion. In case of Yersinae spp. the protein responsible for adhesion and invasion is invasin, an adhesive protein encoded by the invA gene on the bacterial chromosome (Atkinson and Williams 2016; Chauhan et al. 2016). Invasin is expressed at the bacterial surface and binds to cellular $\beta 1$ integrins with a much higher affinity than natural extracellular matrix ligands, such as fibronectin (Tran Van Nhieu and Isberg 1993). Subsequently, invasin has the ability to evoke dimerization, allowing the clustering of $\beta 1$ integrins on the interacting cell (Dersch and Isberg 1999), which then induces the formation of pseudopods to internalize bacteria (Gillenius and Urban 2015; Niemann et al. 2004). Based on the present finding that LFchimera inhibited bacterial invasion, but not adhesion, apparently mediated by an effect on the HeLa cells, it is hypothesized that the LFchimera specifically influenced the clustering of $\beta 1$-integrins, thereby preventing downstream internalization.

Triggered by the cellular response of LFchimera on invasion the effect on proinflammatory cytokines like IL-8 an IL-6 was monitored. LFchimera induced stimulation of IL-8 expression by Yersinia infected cells and exhibited a direct effect on the stimulation of IL-6 expression, independent from Yersinia infection (Fig. 5). In this respect it is interesting that the level of IL-8 secreted by HeLa cells in response to invasion by $Y$. entrocolitica, was significantly lower with virulent strains than with non-virulent strains (Schulte et al. 1996, 2000). Of course further experimental data are needed to elucidate the underlying mechanism. The effects of bLF on the intracellular survival of bacterial pathogens and the differential modulation of the inflammatory response of epithelial models (Frioni et al. 2014) may be a lead but also shows that the mechanism is very complex and strongly depends on the system used (Kim et al. 2012; Sessa et al. 2017).

In conclusion, this research shows that LFchimera has the capacity to inhibit host-cell invasion by Yersiniae spp. and that this effect is host-cell mediated and not bacteria mediated. The mechanism might be linked to effect of invasion on $\beta 1$ integrins but remains to be further investigated. Exposure of the host cells to both LFchimera and the bacterial strains elicits a proinflammatory cytokine release from the cells.
Acknowledgements This work was financially supported by the Dutch Ministry of Defense (Program V1036). We kindly thank Hugo-Jan Jansen and Ruud Busker for their advice.

Open Access This article is distributed under the terms of the Creative Commons Attribution 4.0 International License (http:// creativecommons.org/licenses/by/4.0/), which permits unrestricted use, distribution, and reproduction in any medium, provided you give appropriate credit to the original author(s) and the source, provide a link to the Creative Commons license, and indicate if changes were made.

\section{References}

Adducci BA, Gruszewski HA, Khatibi PA, Schmale DG (2016) Differential detection of a surrogate biological threat agent (Bacillus globigii) with a portable surface plasmon resonance biosensor. Biosens Bioelectron 78:160-166

Atkinson S, Williams P (2016) Yersinia virulence factors-a sophisticated arsenal for combating host defences. F1000Research. https://doi.org/10.12688/f1000research. 8466.1

Bancerz-Kisiel A, Szweda W (2015) Yersiniosis-a zoonotic foodborne disease of relevance to public health. Ann Agric Environ Med 22:397-402

Bikker FJ, Kaman-van Zanten WE, de Vries-van de Ruit AM, Voskamp-Visser I, van Hooft PA, Mars-Groenendijk RH, de Visser PC, Noort D (2006) Evaluation of the antibacterial spectrum of drosocin analogues. Chem Biol Drug Des 68:148-153

Bolscher JGM, Adão R, Nazmi K, van den Keybus PAM, van 't Hof W, Nieuw Amerongen AV, Bastos M, Veerman ECI (2009) Bactericidal activity of LFchimera is stronger and less sensitive to ionic strength than its constituent lactoferricin and lactoferrampin peptides. Biochimie 91:123-132

Bolscher J, Nazmi K, van Marle J, van 't Hof W, Veerman E (2012) Chimerization of lactoferricin and lactoferrampin peptides strongly potentiates the killing activity against Candida albicans. Biochem Cell Biol 90:378-388

Chauhan N, Wrobel A, Skurnik M, Leo JC (2016) Yersinia adhesins: an arsenal for infection. Proteom Clin Appl 10:949-963

Dawson RM, Liu CQ (2008) Properties and applications of antimicrobial peptides in biodefense against biological warfare threat agents. Crit Rev Microbiol 34:89-107

Dersch P, Isberg RR (1999) A region of the Yersinia pseudotuberculosis invasin protein enhances integrin-mediated uptake into mammalian cells and promotes self-association. EMBO J 18:1199-1213

Di Biase AM, Tinari A, Pietrantoni A, Antonini G, Valenti P, Conte MP, Superti F (2004) Effect of bovine lactoferricin on enteropathogenic Yersinia adhesion and invasion in HEp-2 cells. J Med Microbiol 53:407-412

Flores-Villaseñor H, Canizalez-Román A, Reyes-Lopez M, Nazmi K, de la Garza M, Zazueta-Beltrán J, León-Sicairos N, Bolscher JGM (2010) Bactericidal effect of bovine lactoferrin, LFcin, LFampin and LFchimera on antibiotic- 
resistant Staphylococcus aureus and Escherichia coli. Biometals 23:569-578

Flores-Villaseñor H, Canizalez-Román A, de la Garza M, Nazmi K, Bolscher JGM, León-Sicairos N (2012a) Lactoferrin and lactoferrin chimera inhibit damage caused by enteropathogenic Escherichia coli in HEp-2 cells. Biochimie 94:1935-1942

Flores-Villaseñor H, Canizalez-Román A, Velazquez-Roman J, Nazmi K, Bolscher JGM, Leon-Sicairos N (2012b) Protective effects of lactoferrin chimera and bovine lactoferrin in a mouse model of enterohaemorrhagic Escherichia coli O157:H7 infection. Biochem Cell Biol 90:405-411

Frioni A, Conte MP, Cutone A, Longhi C, Natalizi T, Marazzato M, Lepanto MS, Valenti P, Berlutti F (2014) Lactoferrin differently modulates the inflammatory response in epithelial models mimicking human inflammatory and infectious diseases. Biometals 27:843-856

Galindo CL, Rosenzweig JA, Kirtley ML, Chopra AK (2011) Pathogenesis of $Y$. enterocolitica and $Y$. pseudotuberculosis in human Yersiniosis. J Pathog. https://doi.org/10.4061/ 2011/182051

Gillenius E, Urban CF (2015) The adhesive protein invasin of Yersinia pseudotuberculosis induces neutrophil extracellular traps via $\beta 1$ integrins. Microbes Infect 17:327-336

Guiyoule A, Rasoamanana B, Buchrieser C, Michel P, Chanteau S, Carniel E (1997) Recent emergence of new variants of Yersinia pestis in Madagascar. J Clin Microbiol 35:2826-2833

Guiyoule A, Gerbaud G, Buchrieser C, Galimand M, Rahalison L, Chanteau S, Courvalin P, Carniel E (2001) Transferable plasmid-mediated resistance to streptomycin in a clinical isolate of Yersinia pestis. Emerg Infect Dis 7:43-48

Haney EF, Nazmi K, Bolscher JGM, Vogel HJ (2012) Structural and biophysical characterization of an antimicrobial peptide chimera comprised of lactoferricin and lactoferrampin. BBA 1818:762-775

Hernandez E, Girardet M, Ramisse F, Vidal D, Cavallo JD (2003) Antibiotic susceptibilities of 94 isolates of Yersinia pestis to 24 antimicrobial agents. J Antimicrob Chemother 52:1029-1031

Hinnebusch BJ, Rosso ML, Schwan TG, Carniel E (2002) Highfrequency conjugative transfer of antibiotic resistance genes to Yersinia pestis in the flea midgut. Mol Microbiol 46:349-354

Hwang PM, Zhou N, Shan X, Arrowsmith CH, Vogel HJ (1998) Three-dimensional solution structure of lactoferricin $\mathrm{B}$, an antimicrobial peptide derived from bovine lactoferrin. Biochem 37:4288-4298

Jansen HJ, Breeveld FJ, Stijnis C, Grobusch MP (2014) Biological warfare, bioterrorism, and biocrime. Clin Microbiol Infect 20:488-496

Kaman WE, Hawkey S, van der Kleij D, Broekhuijsen MP, Silman NJ, Bikker FJ (2011) A comprehensive study on the role of the Yersinia pestis virulence markers in an animal model of pneumonic plague. Folia Microbiol 56:95-102

Kanthawong S, Nazmi K, Wongratanacheewin S, Bolscher JGM, Wuthiekanun V, Taweechaisupapong S (2009) In vitro susceptibility of Burkholderia pseudomallei to antimicrobial peptides. Int $\mathbf{J}$ Antimicrob Agents 34:309-314
Kim CW, Lee TH, Park KH, Choi SY, Kim J (2012) Human lactoferrin suppresses TNF- $\alpha$-induced intercellular adhesion molecule- 1 expression via competition with NF- $\kappa \mathrm{B}$ in endothelial cells. FEBS Lett 586:229-234

León-Sicairos N, Angulo-Zamudio UA, Vidal JE, López-Torres CA, Bolscher JGM, Nazmi K, Reyes-Cortes R, ReyesLópez M, de la Garza M, Canizalez-Román A (2014) Bactericidal effect of bovine lactoferrin and synthetic peptide lactoferrin chimera in Streptococcus pneumoniae and the decrease in luxS gene expression by lactoferrin. Biometals 27:969-980

Longhi C, Conte MP, Bellamy W, Seganti L, Valenti P (1994) Effect of lactoferricin B, a pepsin-generated peptide of bovine lactoferrin, on Escherichia coli HB101 (pRI203) entry into HeLa cells. Med Microbiol Immunol 183:77-85

Niemann HH, Schubert WD, Heinz DW (2004) Adhesins and invasins of pathogenic bacteria: a structural view. Microbes Infect 6:101-112

Oyston PC, Williamson ED (2013) Prophylaxis and therapy of plague. Expert Rev Anti Infect Ther 11:817-829

Puknun A, Bolscher JGM, Nazmi K, Veerman EC, Tungpradabkul S, Wongratanacheewin S, Kanthawong S, Taweechaisupapong S (2013) A heterodimer comprised of two bovine lactoferrin antimicrobial peptides exhibits powerful bactericidal activity against Burkholderia pseudomallei. World J Microbiol Biotechnol 29:1217-1224

Puknun A, Kanthawong S, Anutrakunchai C, Nazmi K, Tigchelaar W, Hoeben KA, Veerman ECI, Bolscher JGM, Taweechaisupapong S (2016) Ultrastructural effects and antibiofilm activity of LFchimera against Burkholderia pseudomallei. World J Microbiol Biotechnol 32:33

Rotz LD, Khan AS, Lillibridge SR, Ostroff SM, Hughes JM (2002) Public health assessment of potential biological terrorism agents. Emerg Infect Dis 8:225-230

Schulte R, Wattiau P, Hartland EL, Robins-Browne RM, Cornelis GR (1996) Differential secretion of interleukin- 8 by human epithelial cell lines upon entry of virulent or nonvirulent Yersinia enterocolitica. Infect Immun 64:2106-2113

Schulte R, Grass GA, Preger S, Fessele S, Jacobi CA, Schaller M, Nelson PJ, Autenrieth IB (2000) Yersinia enterocolitica invasin protein triggers IL-8 production in epithelial cells via activation of Rel p65-p65 homodimers. FASEB J 14:1471-1484

Sessa R, Di Pietro M, Filardo S, Bressan A, Rosa L, Curone A, Frioni A, Berlutti F, Paesano R, Valenti P (2017) Effect of bovine lactoferrin on Chlamydia trachomatis infection and inflammation. Biochem Cell Biol 95:34-40

Sijbrandij T, Ligtenberg AJM, Nazmi K, Veerman ECI, Bolscher JGM, Bikker FJ (2017) Effects of lactoferrin derived peptides on simulants of biological warfare agents. World J Microbiol Biotechnol 33:3

Tan Y, Liu W, Zhang Q, Cao S, Zhao H, Wang T, Qi Z, Han Y, Song Y, Wang X, Yang R, Du Z (2017) Yersinia pestis YopK inhibits bacterial adhesion to host cells by binding to the extracellular matrix adaptor protein Matrilin-2. Infect Immun 19:85

Tran Van Nhieu G, Isberg RR (1993) Bacterial internalization mediated by beta 1 chain integrins is determined by ligand affinity and receptor density. EMBO J 12:1887-1895 
Verma SK, Tuteja U (2016) Plague vaccine development: current research and future trends. Front Immunol 14:602

Wang X, Qiu H, Jin D, Cui Z, Kan B, Xiao Y, Xu Y, Xia S, Wang H, Yang J, Wang X, Hu W, Xu J, Jing H (2008) O:8 serotype Yersinia enterocolitica strains in China. Int J Food Microbiol 125:259-266 